\title{
Optimised analysis of deuterium depth profiles in beryllium
}

\author{
M. Oberkofler, \\ R. Piechoczek \\ and Ch. Linsmeier \\ Max-Planck-Institut für Plasmaphysik, EURATOM Association, \\ Boltzmannstraße 2, 85748 Garching b. München, Germany
}


Abstract. Various accelerator-based ion beam analysis techniques are reviewed in simulations as well as experimentally with emphasis on their depth resolution and sensitivity for detecting deuterium in beryllium. The depth information contained in the $\alpha$ particles that originate from the nuclear reaction analysis (NRA) with ${ }^{3} \mathrm{He}$ is compared to elastic recoil detection analysis (ERDA) with various projectiles. The best depth resolution for D implanted up to approximately $150 \mathrm{~nm}$ below the Be surface is obtained with medium-heavy ion ERDA: For ERDA with $10 \mathrm{MeV}^{28} \mathrm{Si}$ ions a resolution of $47 \mathrm{~nm}(2 \sigma)$ is experimentally attained. ERDA with ${ }^{4} \mathrm{He}$ and ${ }^{28} \mathrm{Si}$ as well as NRA are applied to analyse a Be sample implanted with $3 \times 10^{22} \mathrm{D} \mathrm{m}^{-2}$ at an energy of $3 \mathrm{keV}$ per atom. The resulting depth profile can only be resolved with medium-heavy ion ERDA. The depth profile of the D concentration features a plateau close to the sample surface with a constant D atomic fraction of 0.1. It drops to zero around a depth of $150 \mathrm{~nm}$, corresponding to the calculated ion range.

PACS codes: 28.52.Fa, 68.55.Ln, 79.20.Rf, 25.55.Ci, 34.50.Bw 


\section{Motivation}

It is widely assumed that deuterium implanted into $\mathrm{Be}$ is retained within the ion penetration range $[1,2,3,4,5,6]$. For keV ions this corresponds to a depth in the range of $100 \mathrm{~nm}$. Numerous experimental findings are in accordance with this assumption: Nuclear reaction analysis (NRA) after implantation reveals no evident lateral diffusion of implanted D; implantation of D at high fluences leads to a saturation of the retained areal density; the desorption temperatures shift with varied implantation energies (and, therefore, with implantation depth); the retained amount at high fluences increases with implantation energy (indicating saturation within the ion range). Direct depth profiling of implanted D has been performed with secondary ion mass spectrometry [7]. However, this method is destructive and has known limitations regarding quantification as well as the calibration of the depth scale. Accelerator-based ion beam analysis (IBA) on the other hand, constitutes (at low analyzing fluences) a non-destructive method that in principle allows the determination of absolute $\mathrm{D}$ depth profiles (in units of areal density). Often nuclear reaction analysis (NRA) using the $\mathrm{D}\left({ }^{3} \mathrm{He}, \alpha\right) \mathrm{p}$ reaction is successfully employed. However, due to the limitations in depth resolution, in some cases elastic recoil detection analysis (ERDA) is the more appropriate choice for D depth profiling. In this contribution ERDA with various projectile ions as well as ${ }^{3} \mathrm{He}$ NRA are reviewed from the perspective of the capability of these methods to resolve the D distribution within a shallow surface layer. Medium-heavy ion ERDA using $10 \mathrm{MeV}$

${ }^{28} \mathrm{Si}$ ions is then employed to assess the depth profile of $\mathrm{D}$ implanted into Be at high fluences and keV energies. 


\section{Experimental}

\subsection{Sample preparation}

The Be samples used were polished polycrystalline disks with mirror finish, procured by MaTecK GmbH. They have a diameter $10 \mathrm{~mm}$ and a thickness of $0.5 \mathrm{~mm}$. The remaining surface roughness $\left\langle R_{a}>\right.$ of these samples is in the range of $5 \mathrm{~nm}$. The purity of the material is $99.8 \mathrm{wt} \%$. Monoenergetic implantation with $\mathrm{D}$ was performed at room temperature at the 'high current source' at IPP [8]. The energy per atom was $3 \mathrm{keV}$. The accumulated fluence was $3 \times 10^{22} \mathrm{D} \mathrm{m}^{-2}$. After implantation the samples were transferred through air to the IBA chamber.

To determine the sensitivity and depth resolution of the various IBA techniques the apparatus functions for NRA and ERDA (with ${ }^{4} \mathrm{He}$ as well as ${ }^{28} \mathrm{Si}$ ) were measured at the projectile energies later used for the analysis of the implanted Be. Thin aC:D layers produced in the 'PlaQ' setup at IPP [9] by plasma deposition onto single crystalline mirror polished $\mathrm{Si}(001)$ were used for these measurements. No pretreatment of the Si substrate was performed before deposition of the films. The film thickness was determined by ellipsometry to $10 \mathrm{~nm}$.

\subsection{Ion beam analysis}

Ion beam analysis was performed at the 'RKS' apparatus at IPP using the fast ions from a $3 \mathrm{MV}$ tandem accelerator. For NRA ${ }^{3} \mathrm{He}$ at an energy of $690 \mathrm{keV}$ was used. The samples were analysed at normal incidence. The signal from the $\alpha$ particles resulting from the nuclear reaction $\mathrm{D}\left({ }^{3} \mathrm{He}, \alpha\right) \mathrm{p}$ was detected at a scattering angle of $\Theta=102^{\circ}$. A solid state detector with a nominal energy resolution of $11 \mathrm{keV}$ resides at a distance of about $25 \mathrm{~mm}$ from the target. It has a rectangular slit aperture of $1 \mathrm{~mm}$ in width and $7 \mathrm{~mm}$ in height. The solid angle (calibrated using thin layers of $\mathrm{Au}, \mathrm{Rh}, \mathrm{Pd}$, $\mathrm{Ti}$ on 
itrous carbon and $\mathrm{Si}$ ) is $9.16 \pm 0.07 \mathrm{msr}$.

ERDA was performed at an incident angle of $\alpha=75^{\circ}$ with respect to the surface normal. The recoiled D atoms were detected in IBM geometry (i.e. with the sample rotation axis orthogonal to the scattering plane) under a scattering angle of $\Theta=30^{\circ}$. The ERDA detector has a nominal energy resolution of $10 \mathrm{keV}$ and resides at a distance of $24.8 \mathrm{~mm}$ from the target. It has a rectangular slit aperture of $0.35 \mathrm{~mm}$ in width and $1.7 \mathrm{~mm}$ in heigth. The solid angle (calibrated using a H containing calibration target and the reacion $\left.\mathrm{H}\left({ }^{4} \mathrm{He}, \mathrm{H}\right)^{4} \mathrm{He}\right)$ is $0.967 \pm 0.05 \mathrm{msr}$. This detector is equipped with a $\mathrm{Ni}$ stopper foil of $5.10 \mu \mathrm{m}$ thickness for filtering of backscattered projectiles. ${ }^{4} \mathrm{He}^{+}$at $3 \mathrm{MeV}$ and ${ }^{28} \mathrm{Si}^{4+}$ at $10 \mathrm{MeV}$ were used as projectiles. To achieve good depth resolution for $\mathrm{D}$ close to the sample surface low energies are preferable. On the other hand, the recoiled D atoms must have enough energy to traverse the stopper foil. Based on these requirements the projectile energies were adapted to yield D energies (for D originating at the sample surface) of about $1.3 \mathrm{MeV}$.

For a quantitative evaluation of the measured spectra knowledge of the energydependent cross sections in the relevant energy ranges at the given detection angles is required. For NRA as well as for He-ERDA these cross sections have been determined experimentally $[10,11,12,13]$. In the case of Si-ERDA the Rutherford expression for the scattering cross section is used. This choice can be justified using the simple formula established by Bozoian [14]. This formula gives an estimate of the projectile energies up to which the deviation of the actual scattering cross section from the Rutherford expression should be below 5\%. The accuracy of Bozoian's formula was tested by comparison to the energy dependence of cross sections for various elastic recoil scattering reactions, namely $\mathrm{D}\left({ }^{4} \mathrm{He}, \mathrm{D}\right), \mathrm{D}(\mathrm{O}, \mathrm{D}),{ }^{4} \mathrm{He}\left(\mathrm{Si},{ }^{4} \mathrm{He}\right)$ and $\mathrm{H}(\mathrm{Si}, \mathrm{H})$. Since these cross sections are not directly available in the literature, they were derived from 
the respective inverse RBS problems $[15,16]$. The cross sections in the inverse RBS geometries were in turn calculated with SigmaCalc [17]. For all mentioned elastic recoil reactions the energies at which the cross sections begin to deviate from the Rutherford expression are within $20 \%$ of the value predicted by Bozoian's formula. In the case of Si ions impinging on $\mathrm{D}$ this formula yields an energy limit of $26 \mathrm{MeV}$. Consequently, recoiling of $\mathrm{D}$ by $10 \mathrm{MeV}$ Si is considered to be correctly described by the Rutherford expression.

\section{Experiment design with help of RESOLNRA and SIMNRA}

To investigate the depth profile of $\mathrm{D}$ after implantation into Be with IBA, a technique with sufficient depth resolution close to the surface must be employed. The simulation program RESOLNRA $[18,19]$ offers the possibilty to calculate achievable resolutions for various IBA methods. Such calculations were performed for a variety of projectile ions taking into account the geometries given in the RKS apparatus (see figure $1 a$ ). As a result the ability of NRA to resolve the $\mathrm{D}$ distribution in a surface layer of about $100 \mathrm{~nm}$ is expected to be marginal. For ERDA with ${ }^{4} \mathrm{He}$ as the projectile the calculations yield only a slight improvement in depth resolution over NRA. A significant resolution enhancement of up to a factor of three is predicted for ERDA with medium-heavy ions. For heavier projectiles (in figure $1 a$ plotted for ${ }^{40} \mathrm{Ar}$ and above) the resolution degrades again with increasing atomic number of the projectile.

In simulations with SIMNRA $[20,19]$ the sensitivity of the various IBA techniques can be assessed. At $\mathrm{MeV}$ energies the cross section for recoiling $\mathrm{D}$ by ${ }^{4} \mathrm{He}$ is enhanced compared to the respective Rutherford expression due to the influence of nuclear forces $[12,13]$. As shown in figure $1 b$ this leads to a sensitivity for ERDA with ${ }^{4} \mathrm{He}$ which is higher than that for NRA by a factor of about 30 . 
The Rutherford expression for ERDA scales with the square of the nuclear charge of the projectiles. Therefore, the sensitivity increases strongly with increasing atomic number. (Screening of the Rutherford cross sections according to Andersen [21] is taken into account in the simulations.) Furthermore, for projectiles with higher atomic number a greater fraction of the primary energy is deposited close to the surface. This further enhances the sensitivity for D close to the surface. Using ${ }^{28} \mathrm{Si}$ instead of ${ }^{4} \mathrm{He}$ improves the sensitivity by more than a factor of 15 .

\section{Experimental apparatus functions}

The achievable depth resolution with NRA and ERDA employing ${ }^{4} \mathrm{He}$ as well as ${ }^{28} \mathrm{Si}$ was assessed experimentally by analysing $10 \mathrm{~nm}$ thick a-C:D films on Si. For such a thin and smooth layer it can safely be assumed that neither the layer thickness nor surface roughness influence the energy spread of the signal significantly. Instead, the spectra shown in the top row of figure 2 can be considered to represent the apparatus functions: They are properties of the method and the apparatus used and are determined by the experimental conditions. The numbers indicating the widths $(2 \sigma)$ of the peaks are derived from Gaussian fits to the signals [22]. The top right graph in figure 2 shows the apparatus function for different footprints of the analysing beam. Due to the effects of geometrical straggling good energy resolution (i.e. a sharp apparatus function) can only be achieved at small beam widths. Assuming the energy-dependent stopping powers in pure Be, the assessed energy resolutions $\sigma$ can be converted into depth resolutions $\sigma_{d}$. The results are summarized in table 1 . The experimental values are higher than calculated with RESOLNRA. One reason for this is that the projectile energies chosen in the experiments $\left(3 \mathrm{MeV}^{3} \mathrm{He}\right.$ and $\left.10 \mathrm{MeV}{ }^{28} \mathrm{Si}\right)$ are higher than in the calculations (1.9 MeV and 7.2 MeV, respectively). Furthermore, surface roughness - while critical for 
small incident and exit angles of the analyzing beam - is not included in the calculations. The relative differences in resolution correspond to the calculated ones. The resolution for ERDA with ${ }^{28} \mathrm{Si}$ is better than the one for NRA by a factor of three. While standard NRA could not resolve a D depth profile confined to a shallow surface region of the order of $100 \mathrm{~nm}$, this is possible with ERDA employing ${ }^{28} \mathrm{Si}$.

\section{Depth profiling of deuterium in berylium}

The Be sample was implanted with D as described in section 2.1 and analysed with NRA as well as ERDA with ${ }^{4} \mathrm{He}$ and ${ }^{28} \mathrm{Si}$. The resulting spectra are shown in the bottom row in figure 2. In the case of NRA and He-ERDA the peak width is in the same range as the width of the corresponding apparatus function and, therefore, dominated by it. The width of the Si-ERDA spectrum is almost 5 times the width of the apparatus function. Furthermore, it is not of Gaussian shape but features a section with increasing intensity towards lower recoil energies. This increase is due to the dependence of the scattering cross section on inverse projectile energy and indicates a constant concentration over a certain depth interval.

Using heavier and more energetic probing projectiles induces more damage in the analysed samples. For Si-ERDA this effect is counteracted by the greater sensitivity compared to NRA. Nevertheless, the observed D depletion with increasing analyzing fluence was much more pronounced in the case of Si-ERDA: Upon analysis of the implanted Be sample with ${ }^{28} \mathrm{Si}^{4+}$ up to a cumulative charge of $1.5 \mu \mathrm{C}$ almost half the retained D areal density was lost from the analysed region. The depletion was slower with increased diameter and reduced current density of the analyzing beam. This fast depletion must be compared to the reduction of the $\mathrm{D}$ content in the sample by only about $10 \%$ in the NRA measurements with a ${ }^{3} \mathrm{He}$ beam after collecting a charge of 
$16 \mu \mathrm{C}$.

Fitting of the Si-ERDA spectrum with SIMNRA leads to the depth profile depicted in figure 3. Layers of thickness $\sigma_{d}$ were used to construct the simulated target. The resulting depth profile features a constant $\mathrm{D}$ atomic fraction of 0.1 up to a depth of about $100 \mathrm{~nm}$. At greater depths the concentration drops to zero within a total depth of $200 \mathrm{~nm}$. In figure 3 the experimental result is compared to a calculation with SDTrim.SP, in which the maximum allowed D atomic fraction was set to 0.1. Compared to the depth profile from the SIMNRA fit the calculated concentration profile shows a steeper gradient at the end of the ion range. Within the experimentally achieved depth resolution the two profiles coincide well.

From previous investigations the maximum D atomic fraction was estimated to be around 0.2 [1]. A source of uncertainty in the former estimations is the unknown

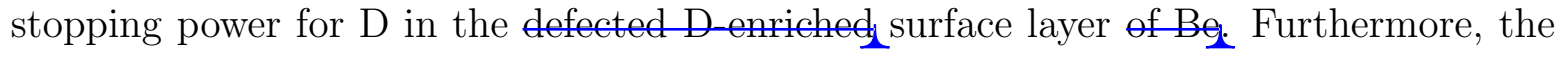
background pressure during implantation was in the range of $10^{-10} \mathrm{mbar}$ in the former investigations. The implantation in the 'high current source' was performed at a pressure of $10^{-7}$ mbar. Oxidation of the Be surface during implantation might therefore influence the retained amount of $\mathrm{D}$. The sample was also transferred through atmosphere from the implantation chamber to the ERDA chamber. The effect of such an exposure to air on the D inventory will be investigated in a future experiment.

\section{Summary and conclusions}

The assets and drawbacks of ERDA with medium-heavy ions compared to He-ERDA and NRA have been adressed. The sensitivity of ERDA is generally higher than for NRA. When ${ }^{4} \mathrm{He}$ is used at $3 \mathrm{MeV}$, ERDA sensitivity is enhanced due to the deviation of the cross section from the Rutherford expression. The ERDA sensitivity increases 
strongly with increasing atomic number of the projectile. However, the impact on the analysed sample also increases, leading to faster depletion compared to lighter projectiles and NRA. For the geometries at the RKS apparatus the depth resolution for D close to the surface in mirror polished Be is $140 \mathrm{~nm}$ for NRA and $116 \mathrm{~nm}$ for He-ERDA. Using ${ }^{28} \mathrm{Si}$ ions for ERDA a substantially improved resolution of $47 \mathrm{~nm}$ can be achieved.

The $\mathrm{D}$ concentration profile after implantation at $3 \mathrm{keV}$ into Be is resolved with ERDA using $10 \mathrm{MeV}^{28} \mathrm{Si}$ ions. At a fluence of $3 \times 10^{22} \mathrm{D} \mathrm{m}^{-2}$ it features a plateau close to the surface and drops to zero in a depth between 100 and $200 \mathrm{~nm}$. This depth profile is in good agreement with results from SDTrim.SP calculations with an upper limit of the $\mathrm{D}$ atomic fraction of 0.1 . This maximum concentration is lower than deduced from previous investigations. Possible reasons for this discrepancy are discussed. The model of trapping of $\mathrm{D}$ in ion-induced traps within the implantation range and accumulation up to a saturation concentration is confirmed by these measurements. 


\section{References}

[1] M. Reinelt, A. Allouche, M. Oberkofler, and Ch. Linsmeier. Retention mechanisms and binding states of deuterium implanted into beryllium. New J. Phys., 11(4):043023, 2009.

[2] A.A. Haasz and J.W. Davis. Deuterium retention in beryllium, molybdenum and tungsten at high fluences. J. Nucl. Mater., 241-243:1076-1081, February 1997.

[3] M. Oberkofler, M. Reinelt, S. Lindig, and Ch. Linsmeier. Structure-dependent deuterium release from ion implanted beryllium: Comparison between Be(1 1 -2 0 ) and Be(poly). Nucl. Instrum. Meth. B, 267(4):718-722, February 2009.

[4] M. Oberkofler, M. Reinelt, A. Allouche, S. Lindig, and Ch. Linsmeier. Towards a detailed understanding of the mechanisms of hydrogen retention in beryllium. Phys. Scr., T138:014036, 2009.

[5] M. Oberkofler, M. Reinelt, and Ch. Linsmeier. Retention and release mechanisms of deuterium implanted into beryllium. Nucl. Instrum. Meth. B, 2011.

[6] R.A. Anderl, R.A. Causey, J.W. Davis, R.P. Doerner, G. Federici, A.A. Haasz, G.R. Longhurst, W.R. Wampler, and K.L. Wilson. Hydrogen isotope retention in beryllium for tokamak plasmafacing applications. J. Nucl. Mater., 273(1):1-26, June 1999.

[7] V.Kh. Alimov, V.N. Chernikov, and A.P. Zakharov. Depth distribution of deuterium atoms and molecules in beryllium implanted with deuterium ions. J. Nucl. Mater., 241-243:1047-1051, February 1997.

[8] W. Eckstein, C. García-Rosales, J. Roth, and W. Ottenberger. Sputtering data. Report IPP 9/82, 1993.

[9] A. Manhard, T. Schwarz-Selinger, and W. Jacob. Quantification of the deuterium ion fluxes from a plasma source. Plasma Sources Sci. T., 20(1):015010, 2011.

[10] W. Möller and F. Besenbacher. A note on the ${ }^{3} \mathrm{He}+\mathrm{D}$ nuclear-reaction cross section. Nucl. Instrum. Meth., 168(1-3):111-114, January 1980.

[11] H.-S. Bosch and G.M. Hale. Improved formulas for fusion cross-sections and thermal reactivities. Nucl. Fusion, 32(4):611, 1992.

[12] A.J. Kellock and J.E.E. Baglin. Absolute cross section for $\mathrm{D}\left({ }^{4} \mathrm{He}, \mathrm{D}\right){ }^{4} \mathrm{He}$ forward scattering. Nucl. Instrum. Meth. B, 79(1-4):493-497, June 1993.

[13] V. Quillet, F. Abel, and M. Schott. Absolute cross section measurements for H and D elastic recoil using 1 to $2.5 \mathrm{MeV}^{4} \mathrm{He}$ ions, and for the ${ }^{12} \mathrm{C}(\mathrm{d}, \mathrm{p}){ }^{13} \mathrm{C}$ and ${ }^{16} \mathrm{O}(\mathrm{d}, \mathrm{p} 1){ }^{17} \mathrm{O}$ nuclear reactions. 
Nucl. Instrum. Meth. B, 83(1-2):47-61, October 1993.

[14] M. Bozoian. A useful formula for departures from Rutherford backscattering. Nucl. Instrum. Meth. B, 82(4):602-603, September 1993.

[15] J.K. Kim, Y.S. Kim, H.W. Choi, G.D. Kim, H.J. Woo, and C.N. Whang. Measurement of elastic recoil cross sections of light nuclei by ${ }^{35} \mathrm{Cl}$ using inverse scattering. Nucl. Instrum. Meth. B, 129(3):323-326, August 1997.

[16] E. Markina, M. Mayer, and H.T. Lee. Measurement of He and H depth profiles in tungsten using ERDA with medium heavy ion beams. Nucl. Instrum. Meth. B, In Press, Accepted Manuscript.

[17] A.F. Gurbich. http://www-nds.iaea.org/sigmacalc/.

[18] M. Mayer. Resolnra: A new program for optimizing the achievable depth resolution of ion beam analysis methods. Nucl. Instrum. Meth. B, 266(8):1852-1857, April 2008.

[19] M. Mayer. http://www.rzg.mpg.de/ mam/.

[20] M. Mayer. Simnra user's guide. Report IPP 9/113, 1997.

[21] H.H. Andersen, F. Besenbacher, P. Loftager, and W. Möller. Large-angle scattering of light ions in the weakly screened Rutherford region. Phys. Rev. A, 21(6):1891, June 1980.

[22] E. Szilagyi, F. Paszti, and G. Amsel. Theoretical approximations for depth resolution calculations in IBA methods. Nucl. Instrum. Meth. B, 100(1):103-121, May 1995. 


\section{Figure captions}

Figure 1

$a$ : Resolution for D in Be close to the surface calculated with RESOLNRA for NRA and ERDA for various projectiles. Energy loss straggling as well as geometrical straggling is included in these calculations. Surface roughness not taken into account. The energy of the projectiles for ERDA was chosen to yield an energy of $1.3 \mathrm{MeV}$ for the recoiled D.

b: Sensitivity for D resulting from SIMNRA calculations for NRA and ERDA for various projectiles. Screened Rutherford cross sections assumed for ERDA. In the case of ${ }^{4} \mathrm{He}$-in ${ }_{\curlywedge}$ addition also the sensitivity resulting from the experimentally determined enhanced (non-Rutherford) cross section is shown. Data points discussed in the manuscript are highlighted in red.

Figure 2

From left to right: Signals from D recorded with NRA, He-ERDA and Si-ERDA. Top row: Analysis of a $10 \mathrm{~nm}$ thick a-C:D layer on Si. Bottom row: Analysis of D-implanted Be. Each spectrum is the sum of several recorded at different positions in order to minimize the effect of depletion during the analysis.

Figure 3

Rectangular columns: D concentration in the target used for the SIMNRA fit to the

Si-ERDA spectrum. Green line: D depth profile from a SDTrim.SP-c $\bar{\equiv}$ llation with the maximum allowed D atomic fraction set to 0.1 .

\section{Table captions}

Table 1

Experimentally achieved resolutions for D in Be for the three methods. 
The energy resolutions $(2 \sigma)$ are converted into depth resolutions $\left(2 \sigma_{d}\right)$ employing the energy_-dependent stopping powers in pure Be. 
Figures

Figure 1.

(a)
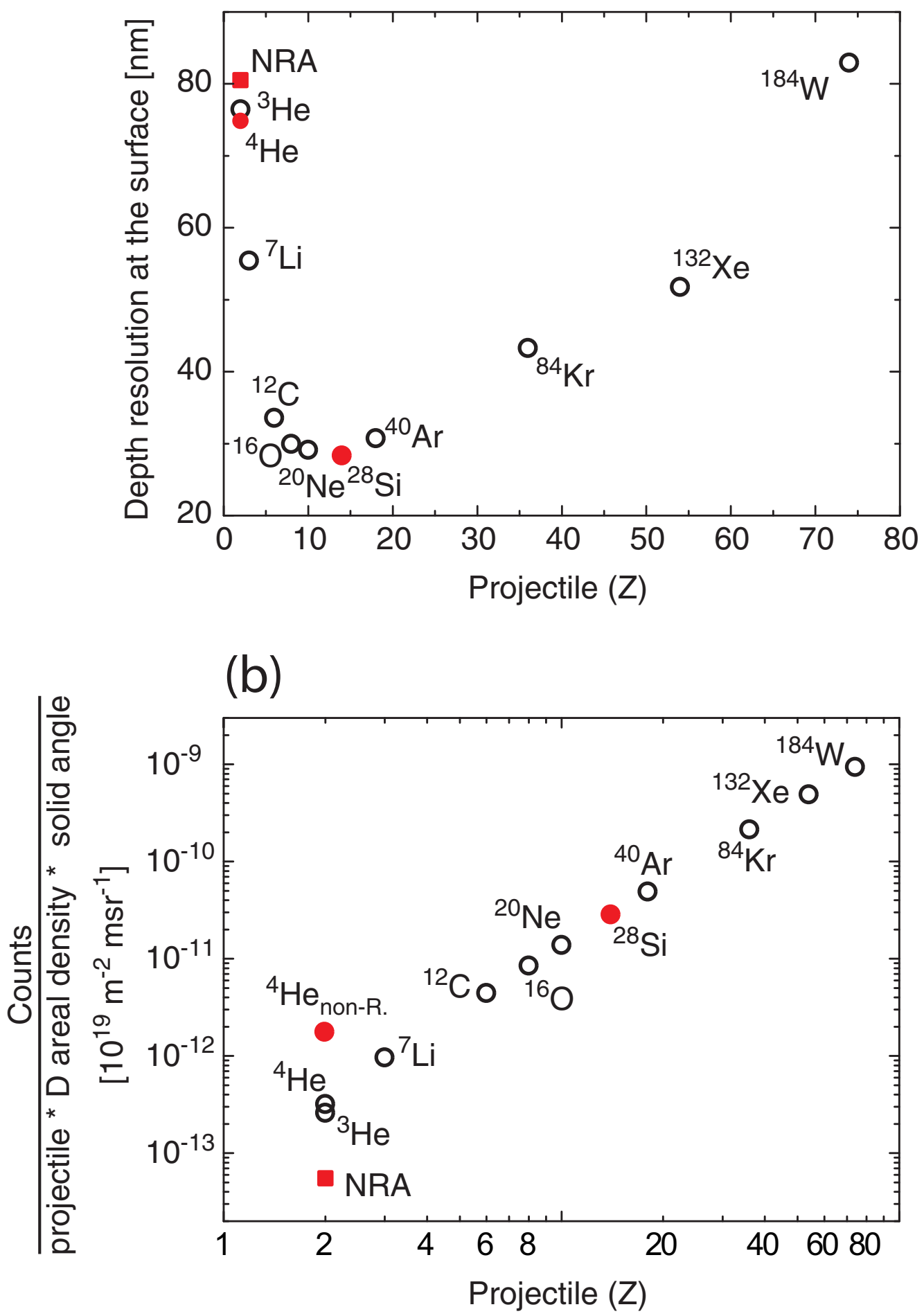
Figure 2.
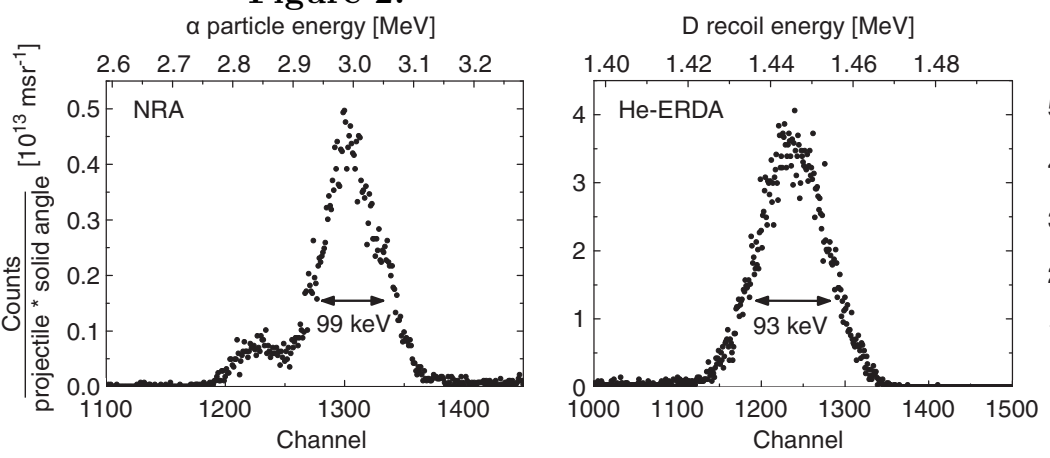

$D$ recoil energy [MeV]
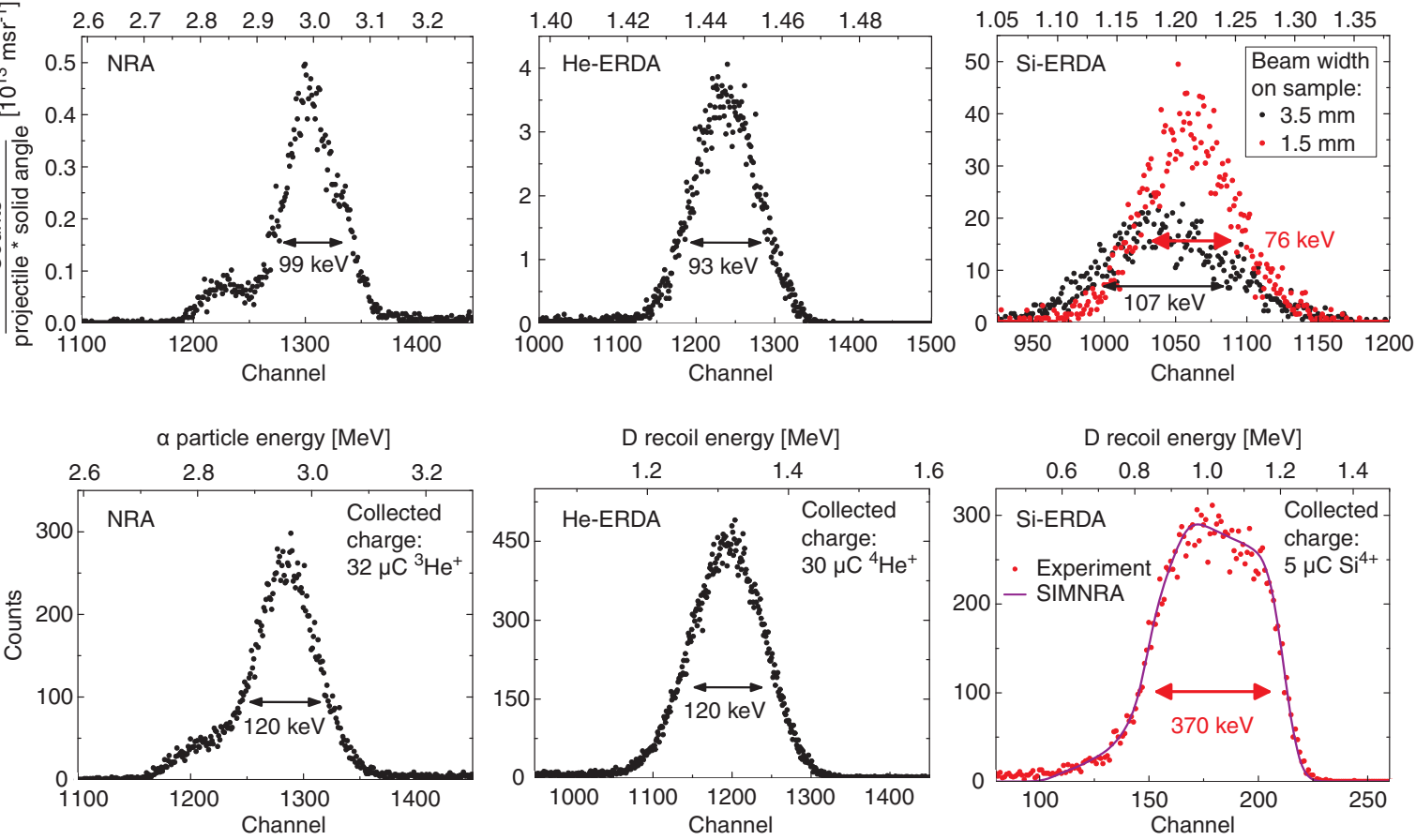
Figure 3.

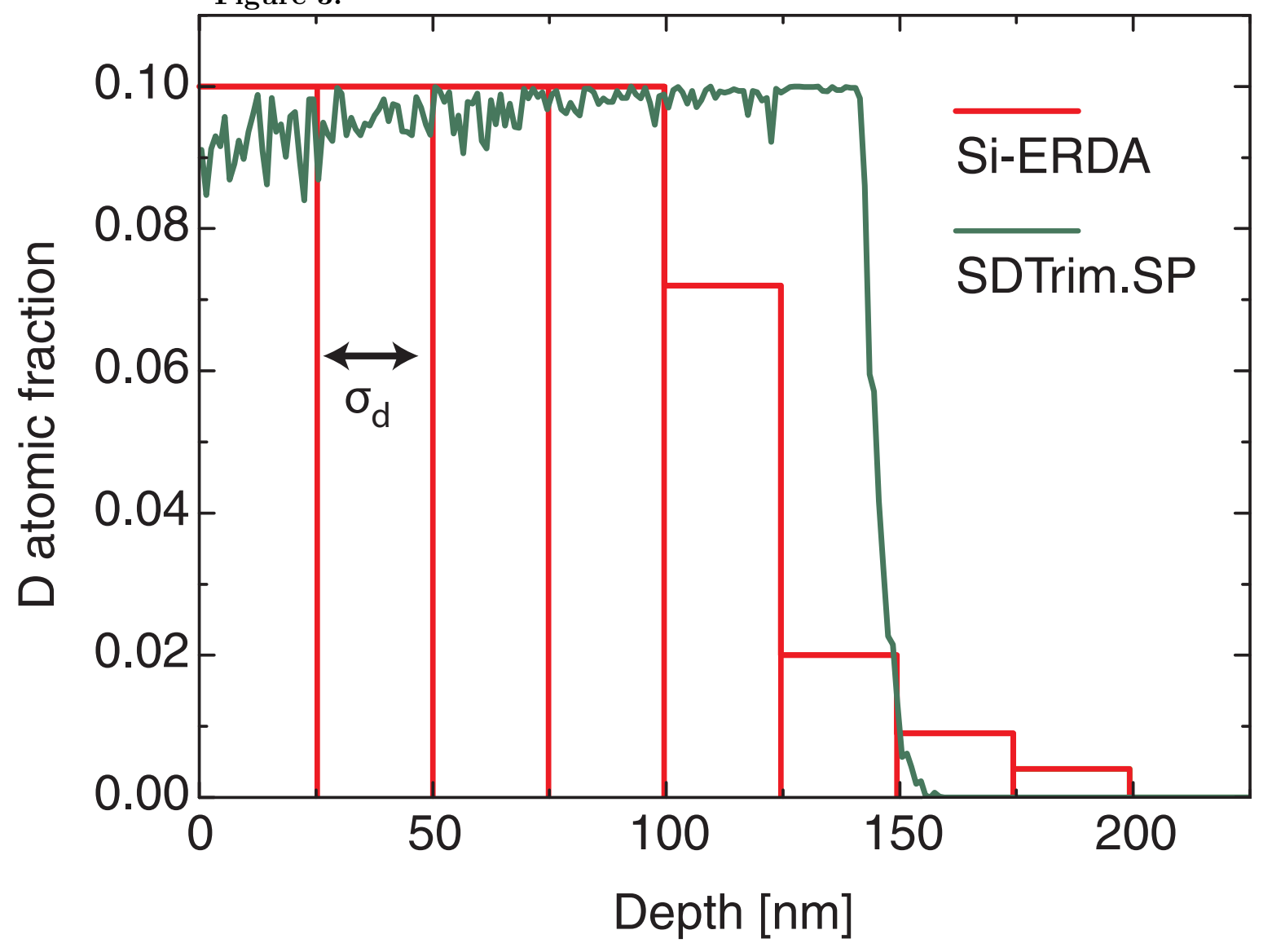




\section{Tables}

\begin{tabular}{|c|c|c|c|}
\hline Method & NRA & He-ERDA & Si-ERDA \\
\hline $2 \sigma[\mathrm{keV}]$ & 99 & 93 & 76 \\
\hline $2 \sigma_{d}[\mathrm{~nm}]$ & 140 & 116 & 47 \\
\hline
\end{tabular}

Table 1. 